\title{
Resección laparoscópica de schwannoma retroperitoneal recurrente (reporte de un caso y revisión de la literatura médica)
}

\section{Laparoscopic resection of a recurrent retroperitoneal schwannoma (case report and review of the literature)}

\section{Sr. Director:}

Presentamos el caso de una mujer de 39 años de edad sin antecedentes de importancia para su padecimiento actual.

Inicia su cuadro hace 6 años con la presencia de dolor lumbar derecho de moderada intensidad.

Se realizan estudios de extensión y se detecta un tumor retroperitoneal derecho, el cual es sometido a resección por cirugía abierta convencional; el reporte histopatológico final es de un schwannoma retroperitoneal benigno escindido en su totalidad.

Cuatro años más tarde, en un estudio tomográfico de control, se encuentra un tumor retroperitoneal derecho de $3 \times 3 \mathrm{~cm}$ adyacente al psoas y a la columna vertebral (fig. 1).

Se decide su resección lumboscópica por reportes previos de schwannomas recurrentes con transformación maligna; el espacio retroperitoneal fue creado mediante una incisión subcostal de $12 \mathrm{~mm}$; se accede al espacio retroperitoneal de manera roma y se introducen $300 \mathrm{~cm}^{3}$ de agua inyectable durante $5 \mathrm{~min}$ a un dedo de guante del número 8 ajustado con seda del 0 a una sonda de Levin número 16; a través de esta apertura se introduce un trocar de $10 \mathrm{~mm}$; se introduce otro de $10 \mathrm{~mm}$, bajo visión directa a nivel del triángulo de Petit, y otro más de $5 \mathrm{~mm}$ a nivel de la línea axilar anterior para triangular ${ }^{1}$. Se realiza la disección del espacio pararrenal y se encuentra un tumor de $3 \times 3 \mathrm{~cm}$ adyacente al psoas, la columna y el riñón, el cual se puede resecar en su totalidad.

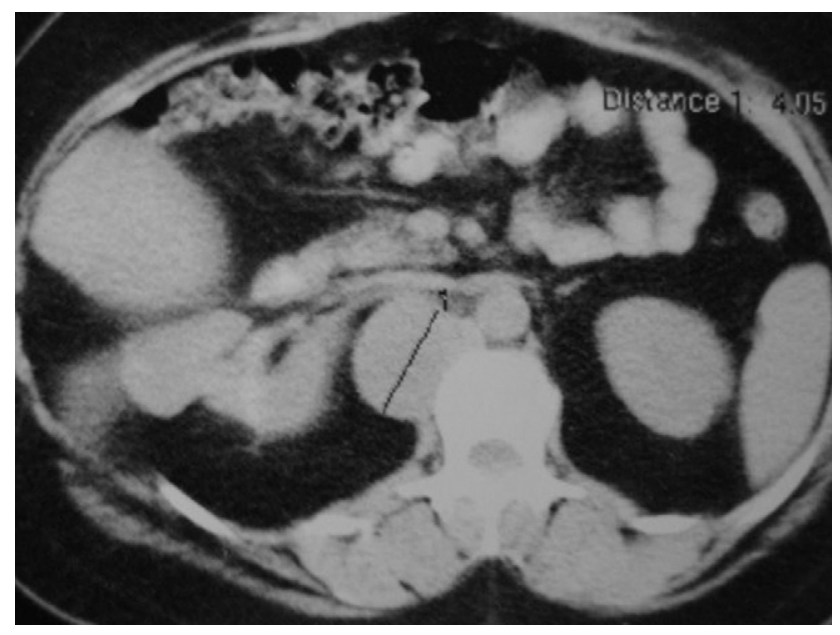

Figura 1 - Schwannoma retroperitoneal.
El reporte histopatológico final fue el de un schwannoma (neurilemoma) benigno retroperitoneal.

Los schwannomas retroperitoneales primarios son tumores raros en ausencia de enfermedad de Von Recklinghausen ${ }^{2}$.

Aproximadamente 50 casos han sido descritos en la literatura médica internacional y una revisión de la India les otorga una prevalencia del 0,5-1,2\% en esta localización ${ }^{3}$. Se trata de un tumor bien encapsulado originado en la vaina periférica del nervio, principalmente de adultos jóvenes; aunque son más comunes en nervios periféricos o pares craneales, localizaciones como el retroperitoneo ya han sido descritas $^{4,5}$. El diagnóstico preoperatorio es difícil, ya que no tiene signos patognomónicos; normalmente se encuentran como hallazgos ante datos de compresión de órganos vecinos o estudios de escrutinio. Aun con el uso de ultrasonido, tomografía o resonancia magnética es imposible asegurar su origen; estos dos últimos ayudan a definir el origen espinal de dichas tumoraciones y su degeneración quística frecuente; se le ha otorgado cierta utilidad a la angiografía o angiorresonancia en ciertos tumores vascularizados, aunque en la mayoría de las ocasiones no es de gran utilidad $^{6}$.

Las biopsias dirigidas normalmente resultan insuficientes o inconclusas, por lo cual el tratamiento quirúrgico ofrece la mejor alternativa para curación o paliación, aunque en general las recurrencias son comunes inclusive en un transcurso menor a 6 meses. La radioterapia ha tenido éxito parcial en los schwannomas benignos, aunque no ofrece beneficio alguno en casos de patología maligna ${ }^{7}$.

En caso de documentarse un crecimiento significativo a partir de la médula espinal, un abordaje combinado con el servicio de Neurocirugía es el más adecuado para garantizar la resección total del tumor ${ }^{8}$.

El diagnóstico patológico se logra al demostrar fascículos fusiformes de células de Schwann con hematoxilina y eosina, y mediante realce inmunohistoquímico a través de proteína S-100.

En el presente caso se utilizó la técnica de retroperitoneoscopia que de manera convencional es realizada en nuestra institución, variante de las descritas previamente por Gaur, Gill y Santinelli. Consideramos que es un abordaje adecuado y seguro para estas recidivas de menor tamaño, logrando una resección amplia y un resultado histopatológico que nos indica la naturaleza de la tumoración.

El retroperitoneo es una localización rara en la presentación del schwannoma; la poca sintomatología y la naturaleza generalmente benigna de la lesión hace que en la mayoría de 
los casos el hallazgo corresponda a una tumoración de gran tamaño, por lo cual la cirugía abierta es la técnica de elección. Sin embargo, en caso de tumores recidivantes o hallazgos incidentales, la resección lumboscópica es una alternativa eficaz para su manejo ${ }^{9,10}$.

B I B L I O G R A F Í A

1. Gaur D. Laparoscopic operative retroperitoneoscopy: Use of a new device. J Urol. 1992;148:1137.

2. Takatera H, Takaha M. Retroperitoneal schwannoma. Urology. 1986;28:529-31.

3. Arshad S, Girish D. Retroperitoneal schwannoma. BHJ. 2002.

4. White HR. Survival in malignant schwannoma. Cancer. 1971;27:720-9.

5. Regan JF, Juler GL, Scmutzer KJ. Retroperitoneal neurilemmoma. Am J Surg. 1977;134:140-5.

6. Goh B, Tan Y, Chung Y, Chow P, Ooi L, Wong W, et al. Retroperitoneal schwannoma. Am J Surg. 2006;192:14-8.

7. Heaton ND, Howard ER. Malignant retroperitoneal schwannoma. J R Soc Med. 1991;84:308-10.
8. Pollo C, Richard A, De Preux J. Résection d'un schwannome retroperitoneal en sablier par abord combiné. Neurochirurgie. 2004;50:53-6.

9. De Diego Rodríguez E, Roca Edreira A, Martín García B, Hernández Rodríguez R, Portillo Martín JA, Gutiérrez Baños JL, et al. Retroperitoneal benign schwannoma. Report of a new case. Actas Urol Esp. 2000;24:685-8.

10. Mir Maresma C, Raventós CX, Celma A, Bestard Vallejo J, Orsola de los Santos A, Morote Robles J, et al. Schwanoma retroperitoneal benigno, exéresis por laparoscopia. Actas Urol Esp. 2008;32:455-7.

P. Ávila Herrera ${ }^{\mathrm{a}, *}$, M. González Domínguez ${ }^{\mathrm{b}}$

O.F. Hernández Ordóñez ${ }^{\mathrm{b}}$ y J. Gutiérrez Aceves ${ }^{\mathrm{a}, \mathrm{b}}$

aServicio de Urología, Nuevo Hospital Civil, Guadalajara, Jalisco, México

${ }^{\mathrm{b}}$ Centro Médico Nacional La Raza, Instituto Mexicano del Seguro Social (IMSS), México, D. F., México

*Autor para correspondencia.

Correo electrónico: bpti@hotmail.com (P. Ávila Herrera).

\section{Duplicación uretral: presentación de un caso clínico y revisión de la literatura médica}

\section{Urethral duplication: case report and review of the literature}

\section{Sr. Director:}

Presentamos el caso de un paciente varón de 7 años de edad, con antecedentes personales de infecciones urinarias de repetición, que consultó por la presencia de un doble orificio a nivel del glande, uno en posición ortotópica y otro más dorsal. Refería la presencia de micciones por ambos orificios, siendo de mayor calibre el chorro procedente del orificio ortotópico, así como un goteo continuo. No relataba historia de instrumentación uretral. Se realizó una ECO renal, que resultó normal, y una cistouretrografía miccional seriada, que mostró una uretra accesoria dorsal completa hasta el cuello vesical (fig. 1). El trayecto principal se correspondía con la uretra ortotópica. Se exploraron ambos meatos uretrales con la introducción de una sonda de $10 \mathrm{Fr}$, observándose un buen paso de esta por los dos meatos. Se realizó una exéresis de la uretra dorsal accesoria, cursando el postoperatorio de forma favorable y con una evolución libre de infecciones urinarias.

La duplicación uretral es una anomalía que afecta mayoritariamente a varones y cuya incidencia es desconocida. Puede acompañarse de otras alteraciones genitourinarias (valvas de uretra posterior, reflujo vesicoureteral, displasia o agenesia renal, extrofia vesical), cardíacas, intestinales (ano imperforado, atresia de esófago), óseas, etc ${ }^{2,3}$. El desarrollo embriológico de la uretra se inicia hacia la tercera semana de gestación con la migración de las células mesenquimales desde la línea primitiva hasta la membrana cloacal originando los llamados pliegues cloacales, de los que derivarán los pliegues uretrales, los cuales hacia el final del tercer mes se cierran a modo tubular sobre la lámina uretral dando lugar a la uretra peneana. Este proceso se completa hacia el cuarto mes con la formación de la uretra glandular. Una noxa en esta

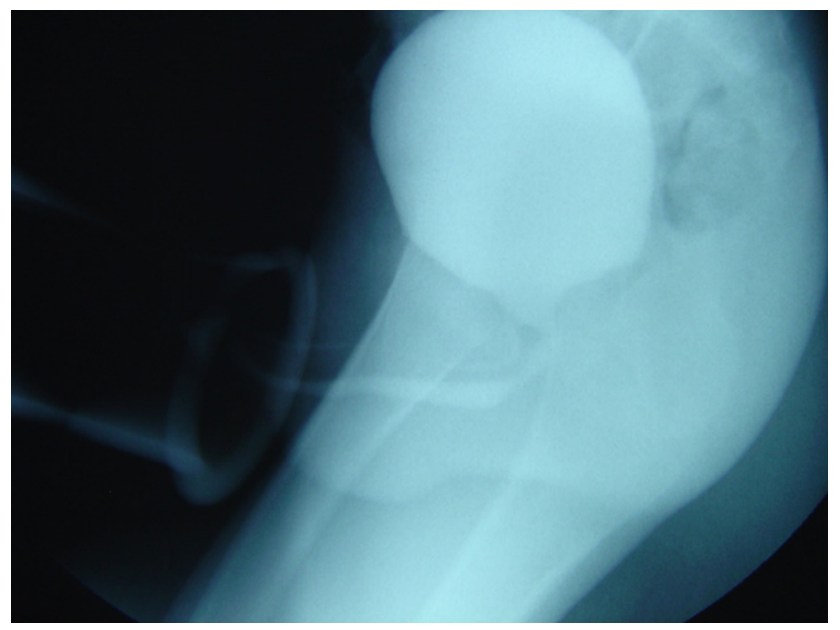

Figura 1 - Cistouretrografía miccional que muestra una uretra accesoria dorsal completa hasta el cuello vesical. 